\title{
Crop Water Availability Mapping in the Danube Basin Based on Deep Learning, Hydrological and Crop Growth Modelling ${ }^{\dagger}$
}

\author{
Silke Migdall ${ }^{1, * \mathbb{D}}$, Sandra Dotzler ${ }^{1}$, Eva Gleisberg ${ }^{1}$, Florian Appel ${ }^{1} \mathbb{D}$, Markus Muerth ${ }^{1}$, Heike Bach ${ }^{1}$, \\ Giulio Weikmann ${ }^{2}$, Claudia Paris ${ }^{2}$, Daniele Marinelli ${ }^{2}$ and Lorenzo Bruzzone ${ }^{2}$ (D) \\ 1 VISTA GmbH, Gabelsbergerstr, 51, 80333 Munich, Germany; dotzler@vista-geo.de (S.D.); \\ gleisberg@vista-geo.de (E.G.); appel@vista-geo.de (F.A.); muerth@vista-geo.de (M.M.); \\ bach@vista-geo.de (H.B.) \\ 2 Department of Information Engineering and Computer Science, University of Trento, Povo, I-38123 Trento, \\ Italy; giulio.weikmann@unitn.it (G.W.); claudia.pari@@unitn.it (C.P.); daniele.marinelli@unitn.it (D.M.); \\ lorenzo.bruzzone@unitn.it (L.B.) \\ * Correspondence: migdall@vista-geo.de; Tel.: +49-89-4521-614-21 \\ + Presented at the 13th EFITA International Conference, online, 25-26 May 2021.
}

check for updates

Citation: Migdall, S.; Dotzler, S.; Gleisberg, E.; Appel, F.; Muerth, M. Bach, H.; Weikmann, G.; Paris, C.; Marinelli, D.; Bruzzone, L. Crop Water Availability Mapping in the Danube Basin Based on Deep Learning, Hydrological and Crop Growth Modelling. Eng. Proc. 2021, 9 , 42. https://doi.org/10.3390/ engproc2021009042

Academic Editors: Dimitrios Aidonis and Aristotelis Christos Tagarakis

Published: 24 January 2022

Publisher's Note: MDPI stays neutral with regard to jurisdictional claims in published maps and institutional affiliations.

Copyright: (C) 2022 by the authors. Licensee MDPI, Basel, Switzerland. This article is an open access article distributed under the terms and conditions of the Creative Commons Attribution (CC BY) license (https:// creativecommons.org/licenses/by/ $4.0 /)$.

\begin{abstract}
The Danube Basin has been hit by several droughts in the last few years. As climate change makes weather extremes and temperature records in late winter and early spring more likely, water availability and irrigation possibilities become more important. In this paper, the crop water demand at field and national scale within the Danube Basin is presented using a dense time series of multispectral Sentinel-2 data, for crop type maps derived with deep learning techniques and physically-based models for crop parameter retrieval and crop growth modelling.
\end{abstract}

Keywords: food security; Thematic Exploitation Platform; cloud processing; crop water demand; crop type classification; biophysical parameter retrieval; water stress; water use efficiency

\section{Introduction}

More droughts, stronger storms, more unpredictable winters-climate change brings more volatile conditions to European agriculture, along with challenges of managing water distribution to fulfil irrigation needs and to support all other ecosystem functions equally. Moreover, growing population and increased food consumption call for measures to stabilise and sustainably heighten the efficiency of agricultural production. To counter some of these trends, data-driven agriculture can provide a new level of information quality for agricultural processes. This is where this study has its target: set within the Horizon 2020 project ExtremeEarth, the issues of water availability and water demand for agricultural production are addressed. The goal is to support irrigation management decisions by combining big data Earth Observation (EO) analysis with water balance and crop growth modelling within European pilot catchments.

In this paper, the first focus is on a wide-scale analysis of the whole Austrian territory as part of the Danube catchment. Its location in the centre of Europe and its different geographic regions are perfect examples of the challenges faced by agriculture and the way big data EO analysis can offer a unique insight into large scale processes and challenges. The second focus is on site-specific analysis for fields in Southern Germany. Cloud Computing and European platform infrastructures were used to pre-process a dense time series of multispectral Sentinel-2 data that were then analysed with a multitemporal deep learning architecture to derive crop type maps. Finally, the water stress of different crops was modelled and the irrigation water demands of various crops were calculated using physically-based models for crop parameter retrieval and crop growth modelling. The results are a big step towards understanding the challenges of water management in the Danube. 


\section{Methods}

\subsection{Satellite Data Pre-Processing}

Figure 1 shows the block-scheme of the proposed method. First, pre-processing of the Sentinel-2 images was conducted on the Food Security TEP, the ESA-supported Thematic Exploitation Platform for agriculture and aquaculture (https: / / foodsecurity-tep. net/, accessed date: 5 January 2022). Its infrastructure allows direct access to all Sentinel-2 satellite imagery and many other datasets. Scalable resources for processing, data handling and storage help to overcome the challenges of big data for large-area EO applications. Within the ExtremeEarth project, a federation with the deep learning platform Hopsworks (https:/ / www.hopsworks.ai, accessed date: 5 January 2022) is being established that allows the exploitation of deep learning approaches for agriculture using the Food Security TEP datasets and resources.

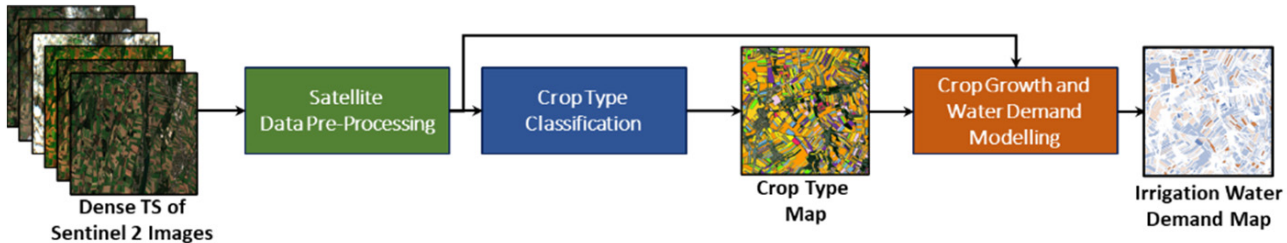

Figure 1. Block-scheme of the system architecture for automatic irrigation water demand mapping.

A dense time series of multi-spectral Sentinel-2 data were the input needed for crop type classification, water stress and water demand. Particularly for the deep learning model, the highest quality atmospheric corrections, including high accuracy cloud and cloud shadow masking in addition to cirrus correction, were necessary. This was offered by VISTA's image processing chains (VIAs), which were implemented as processors on the Food Security TEP. They comprised sophisticated methods for all necessary pre-processing steps in addition to a crop-type independent derivation of plant physiological parameters, so that a time series of high-quality atmospherically corrected multispectral Sentinel-2 data could be delivered for deep learning analysis. Finally, the time series (TSs) were harmonised from the temporal viewpoint by generating a TS of 12 monthly composites for each tile [1]. This regularised the TSs of images acquired on different tiles and mitigated the impact of cloud coverage.

\subsection{Crop Type Classification}

The goal of this step was to automatically produce crop type and crop boundary maps at country scale. To this end, the multitemporal long short-term memory (LSTM) deep learning model was trained by considering the TimeSen2Crop dataset reporting 16 crop types [2]. This publicly available dataset is made up of one million samples collected in Austria for the agronomic year (i.e., period from one year's harvest to the next one) ranging from September 2017 to August 2018. The LSTM deep learning model was trained to generate the crop type map of the whole Austrian cultivated area $\left(\sim 36,000 \mathrm{~km}^{2}\right)$. This multitemporal deep learning model was designed to handle sequential data by classifying the current samples while also exploiting previous ones. The hyper-parameters of the LSTM were selected according to the standard grid-search approach by testing all combinations of the number of network layers $(2,3,4)$ and the number of cells per layer $(100,125,200,225$, 300). The cross-entropy loss was evaluated at each training step, while the optimization problem was addressed by stochastic gradient descent (RMSProp optimiser). The initial learning rate and the weight decay were equal to $10^{-3}$ to 0.4 , respectively. The distributed LSTM was implemented in Hopsworks.

\subsection{Crop Growth and Water Demand Modelling with PROMET}

Based on the crop type classification, a representative sample of pixels $(355,000)$ was distributed over the study area with an overall area of $83.879 \mathrm{~km}^{2}$. Samples were located at 
a certain distance from field boundaries or roads to guarantee pure crop-specific remote sensing information. For each crop, the development of the leaf area, represented by the LAI, was retrieved by inversion of Sentinel-2 time series, for 15 tiles covering $100 \times 100 \mathrm{~km}$ each, using the radiative transfer model SLC [3]. The leaf area information was then assimilated into the crop growth model PROMET [4]. The physically based agro-hydrological model allowed the simulation of, for example, photosynthesis, evapotranspiration, soil moisture, biomass increase, phenological development, and crop water stress, for every sample in hourly temporal resolution.

Crop water stress was indicated as soon as photosynthesis was limited by water shortage and was displayed as a normalised index ranging between 0 (max. stress) and 1 (no stress). Yield in $\mathrm{t} / \mathrm{ha}$ and water use efficiency (WUE) linked yield with water loss through the plant (total evapotranspiration) derived for each season [5]. The model was forced by meteorological parameters (precipitation, air temperature, humidity, radiation and wind speed) extracted from ERA5 reanalysis data (e.g., available from https:/ / cds. climate.copernicus.eu/, accessed date: 5 January 2022).

\section{Results}

\subsection{Simulation Results at Field Scale}

The crop water demand for some fields along the Bavarian-Austrian borders with different crop types such as soybean, corn, winter barley and winter wheat was simulated with the crop growth model PROMET for the year 2018 (Figure 2). In 2018, the soybean fields had the largest crop water demand which was not satisfied by precipitation, while the other crop types had significantly less crop water demand. Crop type assignment is very important for correct quantification of crop-specific irrigation water demand, and it differs from year to year, thus, it is important to simulate it with meteorological data and Earth Observation information.
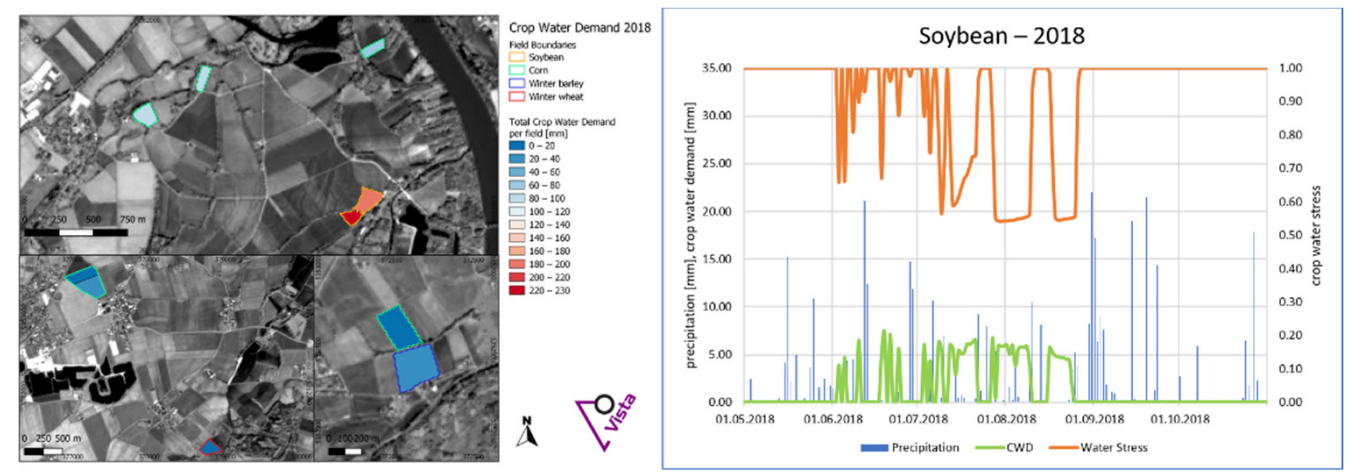

Figure 2. Water demand from different crops of a farm in Southern Bavaria and respective soil moisture and crop water stress time series of a soybean field in the depicted area in 2018.

\subsection{Simulation Results on a National Scale for Austria}

To upscale the approach to derive crop water demand for different crop types on a national scale, a validated crop type map and weather data from 2018 were used for NUTS 2 regions in Austria. Regional differences due to anomalies in spring and summer precipitation affected soil moisture development and crop water stress in the eastern parts of Austria. As a result, severe crop water stress occurred in May and June increasing the simulated irrigation water demand for various crops, especially soybean (Figure 3). Comparison of winter wheat and soybean clearly showed that differences in growing periods and phenology led to differing irrigation water requirements for each crop type, highlighting the need for accurate, high resolution crop type maps for this analysis. 


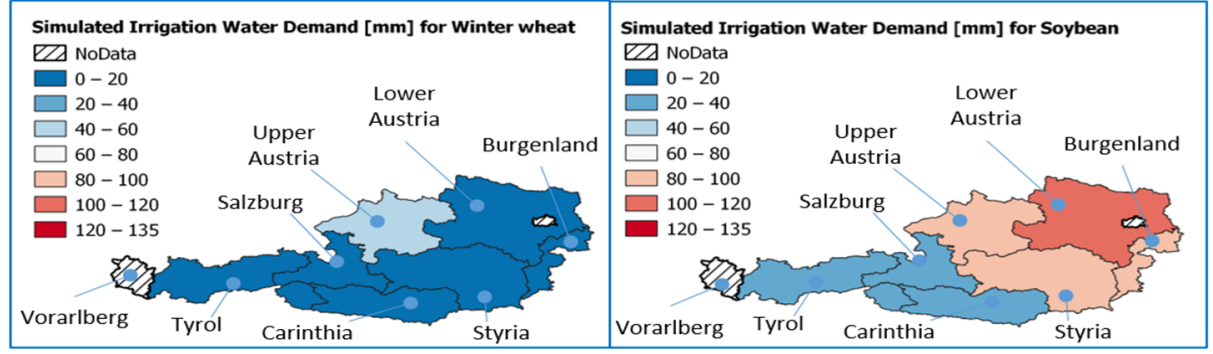

Figure 3. Irrigation water demand in $\mathrm{mm}$ for winter wheat and soybean for Austria in the year 2018.

\section{Conclusions and Outlook}

This work provides a small example of the capabilities of solution-driven methods for water demand calculations making use of Sentinel-2, crop type mapping and crop growth modelling. Using EO (big) data and DL to retrieve detailed crop type information and local phenological and biomass development, and feeding the information into the physical model, enabled the qualification and quantification of the water stress of a variety of crops at farm and regional level in Austria for the extreme year 2018. During ExtremeEarth, the presented methods will be extended to more seasons and regions. In combination with water availability calculations (integrating the hydrological cycle of the catchments) in addition to predictions of water availability in the summer months, these water demand calculations can lead to irrigation policy advice, suitable from local to regional level. EOderived crop information has proven its potential to support and improve crop and water related challenges in future farming and food security management.

Supplementary Materials: The following are available online at https:/ /www.mdpi.com/article/10 .3390/engproc2021009042/s1.

Author Contributions: Conceptualization, S.M., C.P., F.A. and G.W.; methodology, S.M., F.A., M.M., C.P. and G.W.; software, F.A., M.M., G.W. and D.M.; validation, E.G., S.D., C.P., G.W., D.M.: visualisation, E.G. and S.D., C.P. and D.M.; formal analysis, E.G., S.D. investigation, E.G., S.D., C.P., G.W.; resources, C.P., G.W. and M.M.; data curation, C.P., G.W., D.M.; writing, S.M., M.M., C.P., D.M.; supervision, H.B., L.B., S.M. and C.P.; project administration, F.A. and L.B.; funding acquisition, S.M., F.A., H.B. and L.B. All authors have read and agreed to the published version of the manuscript.

Funding: This research was funded by the European Union's Horizon 2020 research and innovation program under grant agreement No. 825258.

Institutional Review Board Statement: Not applicable.

Informed Consent Statement: Not applicable.

Data Availability Statement: The crop classification data is available in a publicly accessible repository. It can be found here: https://rslab.disi.unitn.it/timesen2crop/, accessed date: 5 January 2022.

Acknowledgments: Thank you to Wolfgang Angermair, whose fields have been analysed in this study.

Conflicts of Interest: The authors declare no conflict of interest.

\section{References}

1. Paris, C.; Weikmann, G.; Bruzzone, L. Monitoring of agricultural areas by using Sentinel 2 image time series and deep learning techniques. In Proceedings of the SPIE 2020, Image and Signal Processing for Remote Sensing XXVI, Online Only, 21-25 September 2020; p. 115330K. [CrossRef]

2. Weikmann, G.; Paris, C.; Bruzzone, L. TimeSen2Crop: A Million Labeled Samples Dataset of Sentinel 2 Image Time Series for Crop-Type Classification. IEEE J. Sel. Top. Appl. Earth Obs. Remote Sens. 2021, 14, 4699-4708. [CrossRef]

3. Verhoef, W.; Bach, H. Simulation of hyperspectral and directional radiance images using coupled biophysical and atmospheric radiative transfer models. Remote Sens. Environ. 2003, 87, 23-41. [CrossRef] 
4. Mauser, W.; Bach, H. PROMET_Large scale distributed hydrological modelling to study the impact of climate change on the water flows of mountain watersheds. J. Hydrol. 2009, 376, 362-377. [CrossRef]

5. Probst, E.; Klug, P.; Mauser, W.; Dogaru, D.; Hank, T. Water Use Efficiency of selected crops in the Romanian Plain-Model studies using Sentinel-2 Satellite Images. Sci. Pap. Ser. E Land Reclam. Earth Obs. Surv. Environ. Eng. 2018, 7, $198-208$. 